\title{
STRUCTURAL AND FUNCTIONAL IMPACT OF G2032R MUTATION IN ROS1 - A THEORETICAL PERSPECTIVE
}

\author{
ANISH KUMAR ${ }^{1,2 *}$, SHANTHI $\mathbf{V}^{1}$, RAMANATHAN K ${ }^{1}$ \\ ${ }^{1}$ Department of Biotechnology, School of Bio Science and Technology, VIT University, Vellore, Tamil Nadu, India. ${ }^{2}$ Department of \\ Bioinformatics, School of Bioengineering and Biosciences, Lovely Professional University, Phagwara, Punjab, India. \\ Email: anish.20215@lpu.co.in
}

Received: 11 February 2017, Revised and Accepted: 28 February 2017

ABSTRACT

Objective: Drug resistance is an imperative issue in the treatment of patients with lung cancer. In this work, investigation of the drug resistance mechanism of G2032R mutation in ROS1 is carried out using computational simulation techniques.

Methods: Molecular docking and molecular dynamics (MD) simulation approach have been utilized to uncover the mechanism behind crizotinib resistance in ROS1 at a molecular level. Normal mode analysis was carried out using ElNemo server which examines the movements and conformational changes in the protein structure. ArgusLab, PEARLS, and Autodock were employed for the docking analysis, whereas GROMACS package 4.5.3 was used for MD simulation approach.

Results: The results from our analysis indicates that wild-type ROS1 (Protein Data Bank Code 3ZBF) could be more crucial for the crizotinib binding as it indicates largest binding affinity, minimum number of H-bonds, and higher flexibility than mutant-type ROS1. Moreover, the theoretical basis for the cause of drug insensitivity is the differences in the electrostatic properties of binding site residues between the wild and mutant ROS1 structures. Our analysis theoretically suggests that E-2027 is a key residue responsible for the ROS1 drug selectivity.

Conclusion: Molecular docking and MD simulation results provide an explanation of the resistance caused by G2032R and may give a key clue for the drug design to encounter drug resistance.

Keywords: ROS1, Crizotinib resistance, Molecular docking, Normal mode analysis, Molecular dynamic simulation.

(C) 2017 The Authors. Published by Innovare Academic Sciences Pvt Ltd. This is an open access article under the CC BY license (http://creativecommons. org/licenses/by/4. 0/) DOI: http://dx.doi.org/10.22159/ajpcr.2017.v10i5.17661

\section{INTRODUCTION}

Lung cancer is a malignant form of tumor which is seen in the lung tissues. Most of the tumors are imitative from epithelial cells. The information has proven that among all the cancers, lung cancer ranks second in the occurrence and first in mortality [1]. Since the introduction of ROS1 inhibitors, most particularly, crizotinib has significantly improved survival of the patients. However, prolonged therapy for $>3$ years invariably results in crizotinib resistance $[2,3]$. About $50 \%$ of the resistance arises from the second site mutations such as L1196M, C1156Y, F1174L, and G1269A in anaplastic lymphoma kinase (ALK) and G2032R in ROS1. These mutations consequences in decreased affinity to crizotinib thus result in a poor drug efficacy. ROS1 is an oncogene belongs to the tyrosine kinase receptor family. It was first discovered in sarcoma RNA tumor virus. Rearrangement in the ROS1 gene leads to fusion of a portion of ROS1 that incorporates the tyrosine kinase domain and the proceeding fusion kinase will lead to changes in the cell [4]. ROS1 rearrangements occur in various types of cancers such as non-small cell lung cancer (NSCLC), cholangiocarcinoma, gastric cancer, ovarian cancer, and glioblastoma. Crizotinib resistance is placed on chromosome 6 at position 23 of Homo sapiens, and usually, 2\% of NSCLC cases are due to ROS1 mutations [5,6]. Awad et al. revealed a secondary mutation by performing a biopsy of lung tumor and found that glycine to arginine substitution at codon 2032, i.e., G2032R mutation in the ROS1 kinase domain. Generally, the metastatic process in lung cancer cells makes surgery and radiation complicated to function, and in that case, only chemotherapy is the only option for treatment. Targeted drug therapy is cytostatic (kills only the tumor cells) while the chemotherapeutic agents kill cells apart from cancer cells $[7,8]$. The 5-year endurance rate for NSCLC is $<15 \%$. Crizotinib take actions against ROS1 the with mechanism of adenosine 5 '-triphosphate (ATP) competitive kinase inhibitor, anticipating binding of ATP and ensuing autophosphorylation, which is needed for launching of the enzyme [9]. In spite of the fact that crizotinib has substantiated itself as a productive counter to ROS1-type NSCLC, obtained resistance has made its advantageous impacts impermanent and has risen as a noteworthy barricade for crizotinib [10]. Despite the available reports on resistance by G2032R mutation in ROS1, the critical role of binding site residues flexibility and differences in the electrostatic properties of binding site residues are less well defined [11]. However, how mutation G2032R in ROS1 really presents drug resistance is not surely known. Resistance to crizotinib is a noteworthy general well-being issue as few medications exist to battle this ailment. Along these lines, resistance against even one of the accessible medications is a noteworthy general public health issue. Resistance mechanism of kinase inhibitors has been studied with the help of MD simulation [12]. These circumstances recommend the improvement of novel and more thriving ROS1 inhibitors, particularly for the management of drug resistance NSCLC. Hence, a normal mode analysis (NMA), molecular docking, and molecular dynamics (MD) have been carried out for the wild- and mutant-type ROS1 so as to provide the detailed information on the primary source of crizotinib resistance in NSCLC due to the G2032R mutation.

\section{METHODS}

\section{Data set}

The three-dimensional (3D) structure of wild ROS1 structure (Protein Data Bank [PDB] Code: 3ZBF) was recovered from the crystal structures of the PDB (Brookhaven PDB) for the investigation [13]. The mutant (G2032R) structure was generated using Swiss PDB Viewer [14]. The molecules of the drug were gathered from PubChem, a database kept up in the NCBI [15]. With the assistance of SMILES strings, the 3D structure 
of molecules was developed utilizing CORINA server. All the heteroatom and water molecules were removed before performing MD simulations.

\section{Identification of binding site residues of ROS1}

The ROS1 structure binding site residues were inspected utilizing ligand contact tool (LCT) program [16]. It produces representation twodimensional representations of protein-ligand complex structures from the usual PDB information. The LCT tool distinguishes the binding site residues in their respective structures based on distance calculation. The binding site residues are regarded as in association while one of its molecules is in $0.5 \AA+$ van der Waals radii cutoff. In the present investigation, a complex of mutant structure with crizotinib was submitted into this tool to distinguish the residues of the binding site in the objective proteins.

\section{Flexibility of binding site residues by NMA}

The significant calculation of the atomic movements of residues of binding can be acquired from the mean square fluctuations of the atoms against their regular positions. These estimations can be identified with the B-factor. B-factor investigation gives comprehension of stability, dynamics, and flexibility of amino acids [17]. Protein flexibility assumes a critical part in protein flexibility and designing of the drug molecules [18]. There are different sorts of contacts which require flexibility of certain amino acids. For the comprehension of binding efficiency, amino acid flexibility in drug binding pouch is thought to be a noteworthy parameter. The flexibility loss ruins the binding effect and the other way around [19]. In this manner, we have examined the flexibility of the amino acids, mean square displacement $\left(\mathrm{R}^{2}\right)$ by the ElNemo program [20].

\section{Computation of docking energy}

In the present study, different docking calculations were utilized to compute the binding productivity of ligand with target protein. It is trusted that various docking approaches positively supportive for the removal of errors in the docking computation [21,22]. In particular, ArgusLab docking algorithm and PEARLS algorithm were employed in our analysis for the prediction of crizotinib binding efficiency. The ArgusLab 4.0.1 docking program had been widely approved with docking precision at $\sim 3 \AA$ for root mean square deviation (RMSD) esteem between the anticipated and unique crystallographic posture [23]. The flexible ligand docking of ArgusLab was accessible by depicting the ligand as a torsion tree. Gatherings of reinforced atoms that did not have rotatable bonds were nodes, while torsion was the associations between the nodes. Topology of a torsion tree was an important element impacting proficient docking. For the docking estimations, the scoring strategy AScore (empirical scoring function) was being utilized. AScore depended upon on the total protein-ligand binding efficiency, considering the accompanying commitments: The van der Waals collaboration between the ligand and the protein, the hydrophobic impact, the hydrogen bonds between the ligand and the protein, the impact of deformation, and the impacts of the translational and rotational entropy loss in the binding procedure, individually [24]. The AScore function with the parameters read from the AScore.prm record was utilized to ascertain of binding energies of the subsequent docked structures. The record contained the coefficients for every term in the scoring function.

PEARLS software is used for computing small molecule ligand-protein, ligand-nucleic acid, protein-nucleic acid, and ligand-protein-nucleic acid interaction energies. The PEARLS services were available at http://ang.cz3.nus.edu.sg/cgi-bin/prog/rune.pl. The binding affinity of the wild-type ROS1 complex and mutant-type ROS1 complex was obtained from PEARLS [25]. Finally, Auto Dock 4.2, which was regarded as the greatest docking technique to find the free binding free energy $(\Delta G)$, utilized in our examination. In this docking tool, we utilized semi-flexible docking methods as a part, of which the objective protein was kept inflexible all through the docking and the ligand was kept flexible to investigate self-assertive number of torsional degrees of freedom additionally the six spatial degrees of flexibility crossed by the translational and rotational constraints. AutoDock utilizes Lamarckian genetic algorithm to explore the best conformers. The grid separating was set to $0.375 \AA$, and for every molecule, a most extreme of 10 docking runs were executed. The active site was defined using AutoGrid utility with $126 \times 126 \times 126$ points to cover the entire region occupying the active site residues of ROS1 [26].

\section{MD simulation}

GROMACS is an amazingly fast tool for figuring the non-bonded contacts. It is handed out free of charge covered by the GNU Public license (http://www.gnu.org/). GROMACS version 4.5.3 was utilized to perform simulations of the wild and mutant types of ALK and ROS1 structures [27]. With the assistance of periodic limit conditions and the SPC water display, the protein was solvated in cubic $0.9 \mathrm{~nm}$ [28]. PRODRG server was utilized to produce topology of the ligand. This server utilizes the GROMOS force field for creating topology record and allowing the types of atom. One thousand steps of steepest descent energy minimization were completed for the proteins. System was equilibrated at steady temperature and pressure. Utilizing an atombased close off of $8 \AA$, the non-bonded account was created. The longrange electrostatic communication was taken care of by particle mesh Ewald calculation while obliges bond lengths at their equilibrium cutoff were taken care of by SHAKE calculation [29,30]. Trajectories were accumulated in traj.trr document, and structural investigation was completed at each picoseconds. RMSD, root mean square fluctuation (RMSF), and hydrogen bonds analysis formed were examined with the help of Gromacs utilities, for example, utilities g_rms, g_rmsf, and g_hbond, respectively. In addition, the electrostatic properties for binding sites were also examined using Gromacs utility, g_potential.

\section{RESULTS AND DISCUSSION}

Binding residues analysis

The binding site residues of ROS1 were retrieved from the LCT program by employing the complex structure of ROS1-crizotinib (PDB ID: 3ZBF). The outcome designated a total of 10 amino acid residues, viz., L1951, A1978, L2026, E2027, L2028, M2029, G2032/R2032, D2083, R2083, and L2086 act as binding residues in the ROS1 structure.

\section{Binding residues flexibility by means of NMA}

Binding residue flexibility is a central way to figure out by which drug exerts biological effects. The binding flexibility allows elevated affinity to be achieved between a drug and its target enzyme. To understand the explanation of drug insensitivity by G2032 mutation, we used the program ElNemo to analyze and compare the flexibility of amino acids of both wild and mutants, which are involved in binding with crizotinib. Table 1 depicts the flexibility of amino acids in the drug-binding pocket of both wild and mutants by means of normalized mean square displacement $\left(R^{2}\right)$. We classified out this evidence into three different

Table 1: Evaluation of normalized mean square displacement of drug-binding amino acids of wild and mutant types of ROS1

\begin{tabular}{llll}
\hline S.No. & $\begin{array}{l}\text { Binding } \\
\text { residues }\end{array}$ & $\begin{array}{l}\text { Normalized } \\
\text { mean square } \\
\text { displacement }\left(\mathbf{R}^{2}\right)\end{array}$ & $\begin{array}{l}\text { Normalized } \\
\text { mean square } \\
\text { displacement }\left(\mathbf{R}^{2}\right) \text { in } \\
\text { mutant-type }\left(\mathbf{n m}^{2}\right)\end{array}$ \\
\hline 1 & L1951 & 0.0406 & 0.0370 \\
2 & A1978 & 0.0123 & 0.0164 \\
3 & L2026 & 0.0034 & 0.0052 \\
4 & E2027 & 0.0059 & $0.0059^{*}$ \\
5 & L2028 & 0.0070 & 0.0082 \\
6 & M2029 & 0.0171 & 0.0076 \\
7 & G2032 & 0.0198 & 0.0113 \\
8 & D2033 & 0.0177 & 0.0102 \\
9 & R2083 & 0.0115 & 0.0107 \\
10 & L2086 & 0.0084 & 0.0082 \\
\hline
\end{tabular}

Bold indicates increased flexibility; italics indicates decreased flexibility, *Indicates identical flexibility 
ranges of flexibility. One is the $\mathrm{R}^{2}$ of amino acids in the drug-binding pocket of mutants which is absolutely the same as $\mathrm{R}^{2}$ of the amino acids in the drug-binding pocket of wild named as identical flexibility. The second was the $\mathrm{R}^{2}$ of amino acids in the drug-binding pocket of mutants which is above than $\mathrm{R}^{2}$ of the amino acids in the drug-binding pocket of wild named increased flexibility. And the last is the $\mathrm{R}^{2}$ of amino acids in the drug-binding pocket of mutants which is lesser than $\mathrm{R}^{2}$ of amino acids in the drug-binding pocket of wild named as decreased flexibility. From the above analysis, we figure out that out of ten preferred drug-binding sites, six of the binding amino acids were in the range of decreased flexibility and three of the binding amino acids were in the range of increased flexibility. On the other hand, only one of the binding amino acids was in the range of identical flexibility. This displays that more number of amino acids engaged in the drug-binding pocket of these mutants lost their flexibility due to their occurrence in the range of decreased flexibility. Flexibility of amino acids in drug-binding pocket, in fact, is thought to be a critical parameter to comprehend the binding efficiency. The loss of flexibility hinders the binding effect and the other way around [31]. These confirmations together with $\mathrm{R}^{2}$ data firmly imply that flexibility is critical for the binding of crizotinib with target structure.

\section{Docking studies}

The binding free energies of the wild and mutant types of ROS1-crizotinib complex resolution by ArgusLab 4.0.1 were -8.78 and $-7.73 \mathrm{kcal} / \mathrm{mol}$, respectively. To figure out the authentic analysis of binding affinity, the new trend in the field is the multiple docking algorithms. Finally, the results of the different algorithms were joined to balance the faults in the in-silico prediction. This approach is helpful in the ligand-binding inquiry for improving the anticipation of identifying true ligands. To justify this, binding affinity was also determined using the PEARLS program. The results are shown in Table 2 .

The PEARLS program results showed that binding energies were found to be -8.80 and $-6.60 \mathrm{kcal} / \mathrm{mol}$ for the wild- and mutanttype structures of ROS1-crizotinib complex, respectively. Further, to strengthen the docking experiment, we have used AutoDock in our analysis $[32,33]$. The results showed that binding energies were found to be -7.68 and $-6.08 \mathrm{kcal} / \mathrm{mol}$ for the wild- and mutant-type structures of ROS1-crizotinib complex, respectively. It indicates the average binding energy of wild ROS1-crizotinib complex as $-8.42 \mathrm{kcal} / \mathrm{mol}$ and of mutant ROS1-crizotinib complex as $-6.80 \mathrm{kcal} / \mathrm{mol}$.

The experimental evidence also indicates that glycine at position 2032 is conserved and arginine substitution at this highly conserved residue conferred resistance to ROS1 inhibitors. For instance, crizotinib concentration needed to achieve 50\% enzyme inhibition (Ki) was increased by a factor of 270 for the G2032R mutant kinase as compared with non-mutant ROS1 [34]. Finally, the intermolecular hydrogen bonds in the complex structure were also shown using LIGPLOT to explain the mechanism of crizotinib resistance [35]. The results are shown in Fig. 1. Hydrogen bonds are determined by dashed lines among the atoms involved, while hydrophobic contacts are shown by an arc with spokes radiating facing the ligand atoms they contact. The contacted atoms are delineated shown with spokes radiating back (Fig. 1).

It is clear from the figure that wild ROS1 structure can display two hydrogen bonds whereas mutant ROS1 structure can show only one hydrogen bond with crizotinib. N22 and N23 of crizotinib are convoluted in hydrogen bonding with E-2027 and M-2029, respectively, in the wild ROS1-crizotinib complex. On the other hand, in the mutant structure, N24 of crizotinib is involved in hydrogen bonding with M-2029. There is no hydrogen bond among E-2027 with crizotinib. To establish our prediction, we have also predicted the intermolecular distance utilizing PyMOL tool. The results indicates that distance between crizotinib and E2027 in wild-ROS1 is $3.56 \AA$ Ahereas distance between crizotinib and E2027 in mutant-ROS1 is $10.38 \AA \AA$ (Fig. 2a and b).

Similarly, the distance between crizotinib and M2029 in mutant structure is also increased (Fig. 3a and b).

These alterations may have an effect on the protein function and binding free energy by generating changes in the interaction energy between crizotinib and ROS1 or affecting the conformations of these residues or local rigidity of the structure.

\section{MD simulation}

The MD simulations were executed for the wild and mutant G2032R crizotinib complex employing GROMACS package 4.5.3. RMSD, RMSF, hydrogen bond, and electrostatic potential details were evaluated. RMSD is the similarity between the two structures. It is evident

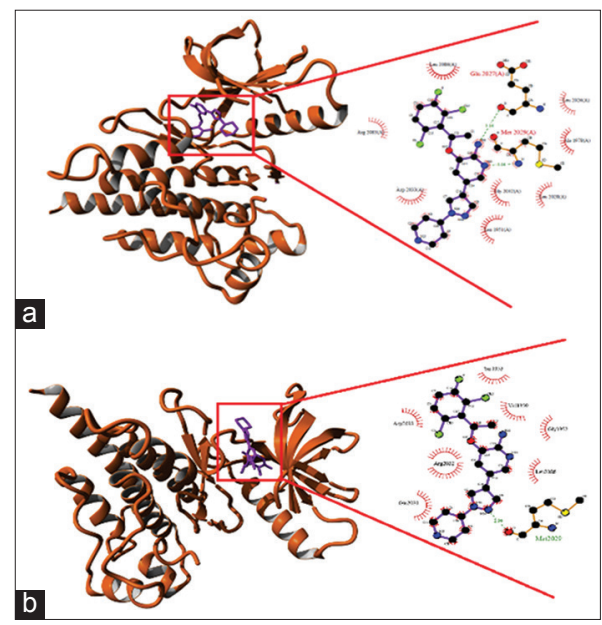

Fig. 1: The three-dimensional model of the active site and contact of crizotinib with (a) wild-type ROS1 (PDB Code 3ZBF) and (b) G2032R mutant type ROS1. Carbon, nitrogen, and oxygen atoms are shown as black, blue, and red balls; hydrogen-bonded and non-bonded protein-ligand contacts are represented as dashed green lines and eyelashes, respectively

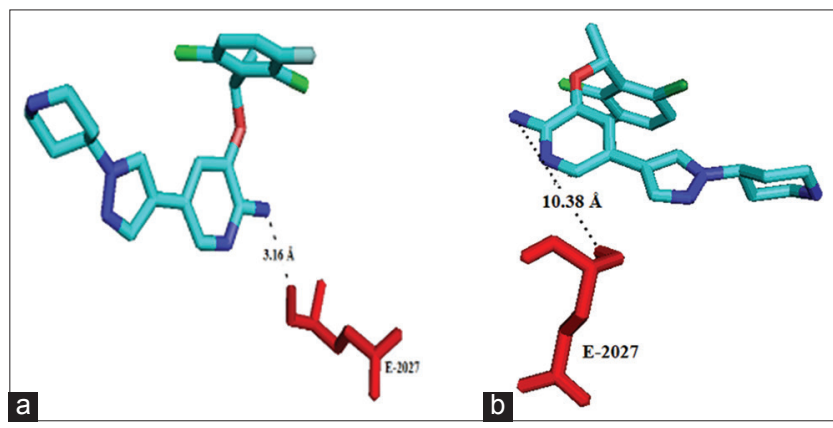

Fig. 2: Distances among crizotinib and E-2027 in (a) wild-type ROS1 and (b) mutant-type ROS1

Table 2: Binding free energy investigation of crizotinib with wild and mutant types of ROS1

\begin{tabular}{llllll}
\hline S.No. & Complex type & $\begin{array}{l}\text { Binding free energy by } \\
\text { "ArgusLab" (kcal/mol) }\end{array}$ & $\begin{array}{l}\text { Binding free energy by } \\
\text { "PEARLS" (kcal/mol) }\end{array}$ & $\begin{array}{l}\text { Binding free energy } \\
\text { by “Autodock" } \\
\text { (kcal/mol) }\end{array}$ & $\begin{array}{l}\text { Average binding free } \\
\text { energy (kcal/mol) }\end{array}$ \\
\hline 1 & Wild-type ROS1-crizotinib & -8.78 & -8.80 & -7.68 & -8.42 \\
2 & Mutant-type ROS1-crizotinib & -7.73 & -6.60 & -6.08 & -6.80 \\
\hline
\end{tabular}


from the Figs. 4 and 5 that both the wild and mutant structures ROS1 have deviated. After the relaxation period, wild structure acquired $\sim 0.32 \mathrm{~nm}$ at $1000 \mathrm{ps}$ during the simulations, while mutant structure acquired $\sim 0.25 \mathrm{~nm}$ of backbone RMSD at 1000 ps (Fig. $4 \mathrm{a}$ ). Between a period of 2000 and 8000 ps, a wild structure is able to maintain $\sim 0.30 \mathrm{~nm}$ of backbone RMSD, while a mutant structure showed frequent changes $(\sim 0.20$ to $\sim 0.28 \mathrm{~nm})$ in backbone RMSD. Wild gained RMSD dominance over mutant and attained RMSD of $\sim 0.35 \mathrm{~nm}$ at 10,000 ps. On the other hand, the mutant showed less deviation than the wild and achieved $\sim 0.24 \mathrm{~nm}$ at $10,000 \mathrm{ps}$. At the end of $15,000 \mathrm{ps}$ (Trail 1), the wild attained RMSD of $\sim 0.30 \mathrm{~nm}$ whereas mutant structure attained RMSD of $\sim 0.27 \mathrm{~nm}$. In Trail 2, at the end of 15,000 ps, the wild attained RMSD of $\sim 0.33 \mathrm{~nm}$ whereas mutant structure attained RMSD of $\sim 0.25 \mathrm{~nm}$. The mutant structure deviated less from the starting structures, but the wild ROS1 showed more deviations. This magnitude of fluctuations together with the very small difference between the average RMSD values concluded that the simulations produced stable trajectories, thus providing a suitable basis for further analysis. Moreover, we have evaluated the flexibility behavior of the amino acid residues (Fig. 4b) in ROS1 by means of RMSF. Analysis of the fluctuations declared that the more degrees of flexibility were observed in the wild than in the mutant ROS1. Most importantly, there was an important variation in the flexibility observed, particularly in the region 1900-2300. The alteration in flexibility is further confirmed

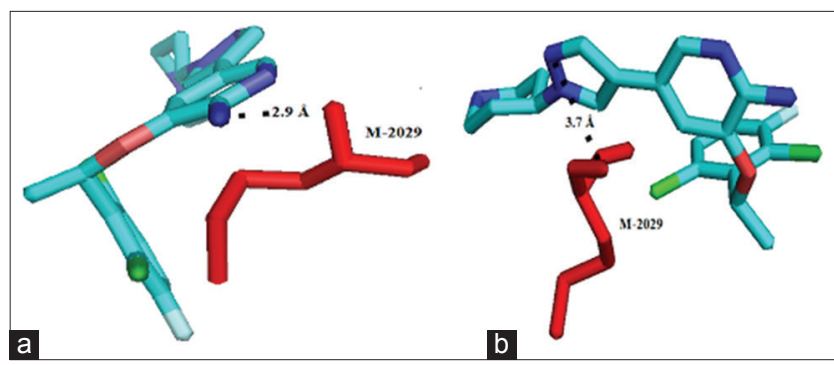

Fig. 3: Distances among crizotinib and M-2029 in (a) wild-type ROS1 and (b) mutant-type ROS1 by the number of the intramolecular hydrogen bond (NH-bond) analysis (Fig. 4c). The analysis showed that mutant gained a slightly higher number of hydrogen bonds when compared to wild structure. During the 1000 ps, the wild structure showed an increase in $\mathrm{H}$ bonds compared to the mutant structure. It can be seen in Fig. 4c; the mutant structure generates $210 \mathrm{H}$ bonds between periods of 3000 and 12,000 ps whereas wild structure generates 202-209 $\mathrm{H}$ bonds between periods of 3000 and 12,000 ps. In Trail 1, at the end of 15,000 ps, the mutant structure generates $212 \mathrm{H}$ bonds whereas wild structure generates 205 H bonds.

In Trail 2, at the end of 15,000 ps, the mutant structure generates $209 \mathrm{H}$ bonds whereas wild structure generates $201 \mathrm{H}$ bonds. The frequency of the occurrence of the $\mathrm{H}$-bond was slightly higher in the mutant structure than the wild structure. These variations within the $\mathrm{H}$-bond networks in ROS1 structure affect the intrinsic flexibility of the protein. On the basis of NH-bond analysis, it is confirmed that the occurrence of the mutation leads to a loss of flexibility in the mutant ROS1 due to the formation of more hydrogen bonds. This enables that the wild structure exhibits stronger binding with crizotinib than the mutant structure.

It is believed that these hydrogen bonds are responsible for the integrity, maintenance, and slightly higher structural stability of the mutant structure than the wild structure. The simulation results also reveal the fact that the binding pocket of mutant enzyme has shown slightly rigid or non-flexible pocket than the binding pocket of wild-type. These behaviors enable the wild structure to exhibit a stronger binding with crizotinib than the mutant structure. Furthermore, to understand the electrostatic properties of wild and mutant structures, we analyzed the charge distribution of hydrophobic pocket residues throughout the simulation box in both wild and mutant structures. The Gromacs make_ndx command is used to select the binding pocket residues. The result is shown in Fig. 6.

It shows that there is an increase of charge in mutant binding pocket residues. The G2032R mutation introduces a charge at this position; this can cause repulsion between the mutated residue and neighboring residues. In addition, the introduction of a positive charge might

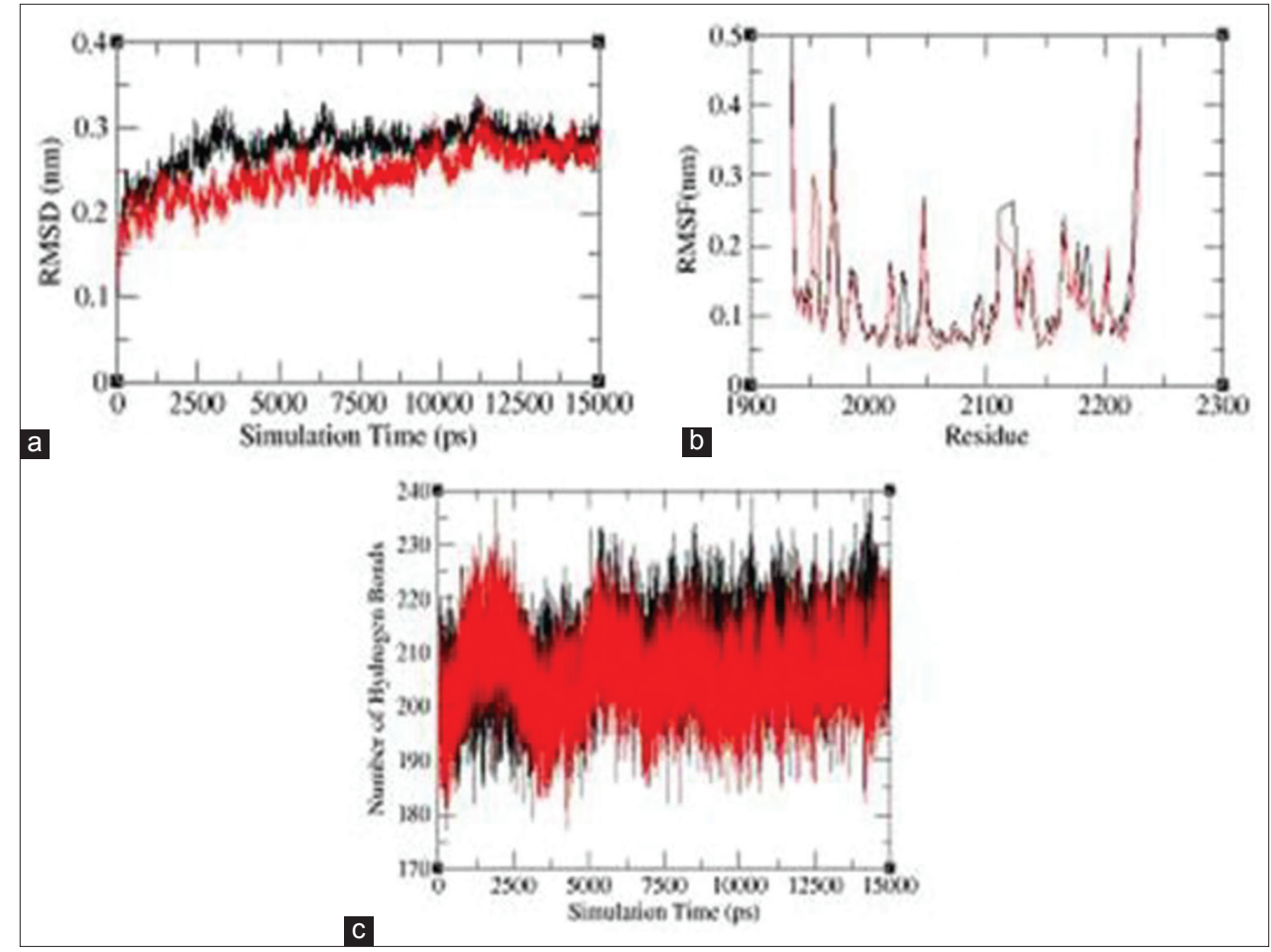

Fig. 4: (a) Root mean square deviation, (b) root mean square fluctuation and (c) NH bond of native and mutant type ROS1 complexes versus time at $300 \mathrm{~K}$. Native is shown in black and mutant is shown in red color 


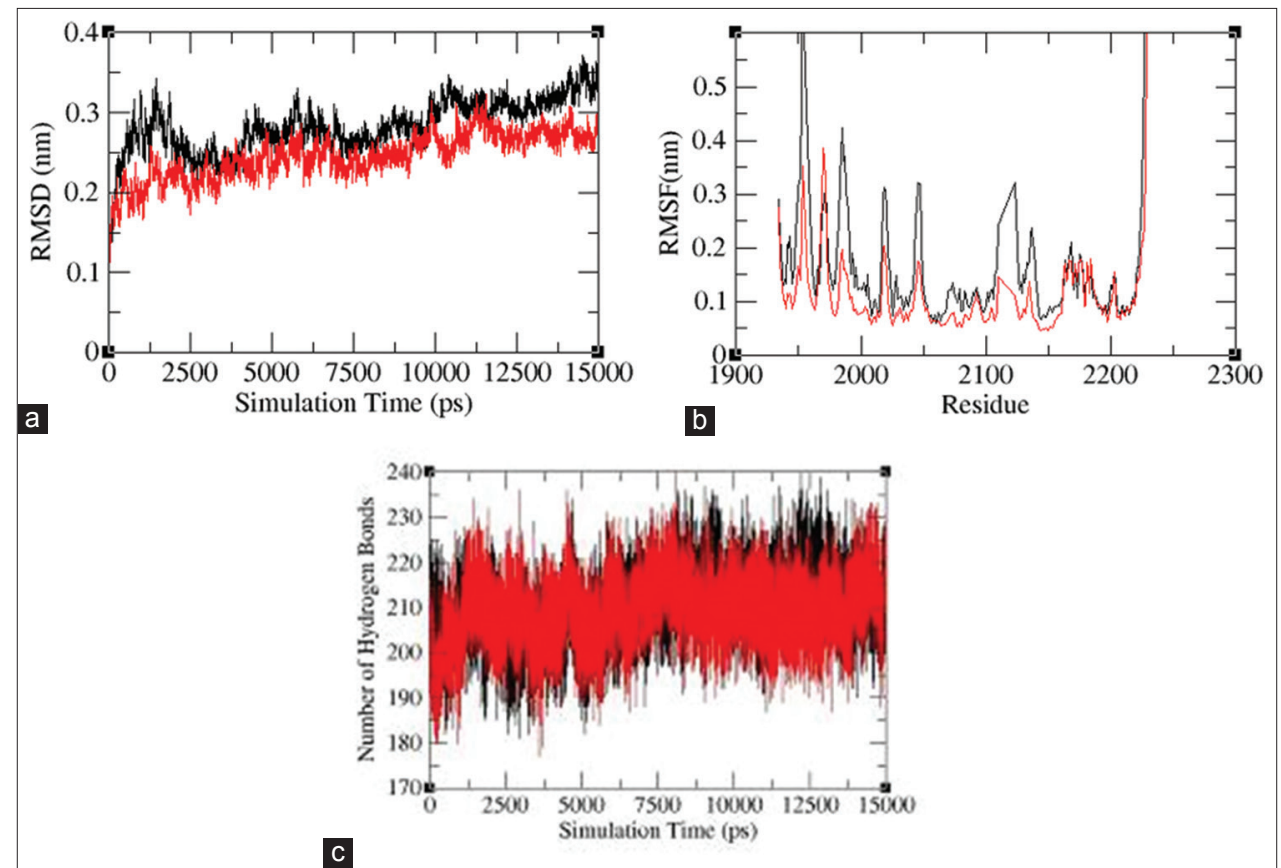

Fig. 5: (a) Root mean square deviation, (b) root mean square fluctuation and (c) NH bond of native and mutant type ROS1 complexes versus time at $300 \mathrm{~K}$. Native is shown in black and mutant is shown in red color

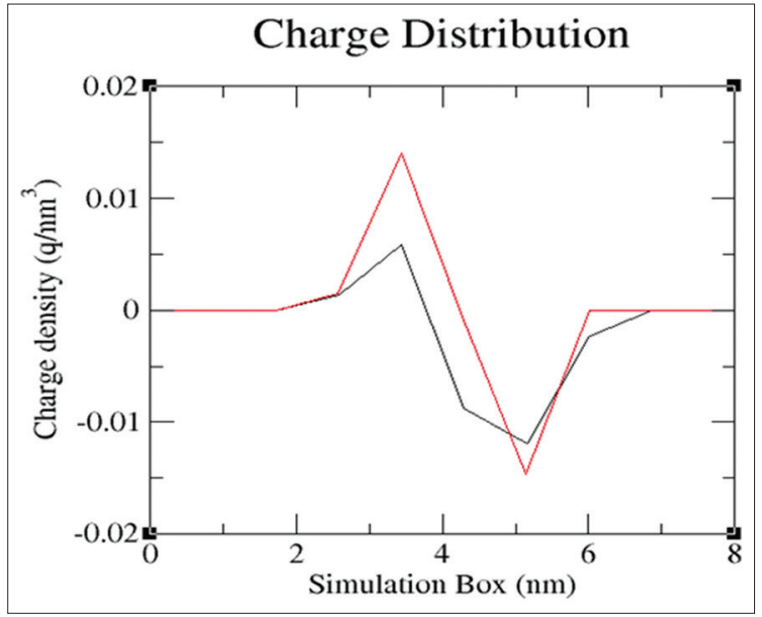

Fig. 6: Charge density analysis of ROS1 along the molecular dynamics simulation. The symbol coding scheme is as follows: Wild (black) and mutant (red)

influence the interactions with the partner molecule. Crizotinib is a targeted form of the drug that specially targets and kills lung cancer cells. Crizotinib resistance becomes a serious issue to lung cancer patients. Although previous findings suggested that drug resistance in ROS1is due to loop conformation changes, in our findings, we observed that intramolecular hydrogen bonds play an important in flexibility. Mutation leads to a loss of flexibility in the mutant ROS1 due to the formation of more intramolecular hydrogen bonds. Glutamic acid residue (E-2027) is absent in the mutant structure, and this residue is important for the effective binding and stability of the ROS1-crizotinib complex. Docking results indicated that mutant ROS1 structure significantly affected the binding affinity for crizotinib. We believe that this could be the important basis for the cause of drug insensitivity.

\section{CONCLUSION}

The advancement in structure prediction techniques and the development of computer simulation and MD have resulted in the increased prominence of rational drug design for finding new and improved drugs. The competence and effect of the rational drug design approach have minimized the drug development time. In this study, we have utilized the NMA and MD way to deal with addition knowledge into crizotinib-resistant G2032R mutation in the structure of ROS1. Detailed information about the molecular and structural properties of the wild and mutant types of ROS1 has been provided by this approach. Docking analysis clearly indicates that G2032R mutation significantly decreases the binding affinity for crizotinib than wild-type ROS1. In addition, the $\mathrm{R}^{2}$ and RMSF data obtained in the molecular simulation approach reveal that the fluctuation behavior of binding residues in the mutant structure is slightly different from the wild structure, and there was an increase in the number of intramolecular hydrogen bonds during MD simulation. This indicates that the G2032R mutation significantly stabilizes the structure of the mutant type ROS1. Our results indicate that glutamic acid residue (E-2027) is most probably needed to make enhanced interactions with crizotinib molecule. Finally, the charge distribution study clearly indicates the loss of hydrophobicity in the binding site due to G2032R mutation. Ideally, the work gives new experiences into the mechanism of drug resistance pattern in lung cancer and put forward a productive idea of devising NSCLC inhibitors.

\section{ACKNOWLEDGMENTS}

Dr. Anish Kumar expresses a deep sense of gratitude to Mr. Rajesh Kumar and Mrs. Neera Rani for constant encouragement provided by them through the research work

\section{REFERENCES}

1. Siegel R, Naishadham D, Jemal A. Cancer statistics, 2012. CA Cancer J Clin 2012;62(1):10-29.

2. Manning G, Whyte DB, Martinez R, Hunter T, Sudarsanam S. The protein kinase complement of the human genome. Science 2002;298(5600):1912-34.

3. Kumar A, Shanthi V, Ramanathan K. Discovery of potential ALK inhibitors by virtual screening approach. 3 Biotech 2016;6:1-12.

4. Christensen JG, Zou HY, Arango ME, Li Q, Lee JH, McDonnell SR, et al. Cytoreductive antitumor activity of PF-2341066, a novel inhibitor of anaplastic lymphoma kinase and c-Met, in experimental models of anaplastic large-cell lymphoma. Mol Cancer Ther 2007;6:3314-22.

5. Roberts PJ. Clinical use of crizotinib for the treatment of non-small cell 
lung cancer. Biologics 2013;7:91-101.

6. Cui JJ, Tran-Dubé M, Shen H, Nambu M, Kung PP, Pairish M, et al. Structure based drug design of crizotinib (PF-02341066), a potent and selective dual inhibitor of mesenchymal-epithelial transition factor (c-MET) kinase and anaplastic lymphoma kinase (ALK). J Med Chem 2011;54(18):6342-63.

7. Shaw AT, Camidge DR, Engelman JA. Clinical activity of crizotinib inadvanced non-small cell lung cancer (NSCLC) harboring ROS1 gene rearrangement. J Clin Oncol 2012:30:7508.

8. Ou SH, Tan J, Yen Y, Soo RA. ROS1 as a 'druggable' receptor tyrosine kinase: Lessons learned from inhibiting the ALK pathway. Expert Rev Anticancer Ther 2012;12(4):447-56.

9. Kumar A, Shanthi V, Ramanathan K. Computational investigation and experimental validation of crizotinib resistance conferred by C1156Y mutant anaplastic lymphoma kinase. Mol Inform 2015;34(23): $105-14$

10. Kumar A, Ramanathan K. Analyzing resistance pattern of non-small cell lung cancer to crizotinib using molecular dynamic approaches. Indian J Biochem Biophys 2015;52(1):23-8.

11. Sun H, Li Y, Tian S, Wang J, Hou T. P-loop conformation governed crizotinib resistance in G2032R-mutated ROS1 tyrosine kinase: Clues from free energy landscape. PLoS Comput Biol 2014;10(7):e1003729.

12. Deng Y, Roux B. Computations of standard binding free energies with molecular dynamics simulations. J Phys Chem B 2009;113(8):2234-46.

13. Berman HM, Westbrook J, Feng Z, Gilliland G, Bhat TN, Weissig H, et al. The protein data bank. Nucleic Acids Res 2000;28(1):235-42.

14. Guex N, Peitsch MC. SWISS-MODEL and the Swiss-PdbViewer: An environment for comparative protein modeling. Electrophoresis 1997;18(15):2714-23

15. Feldman HJ, Snyder KA, Ticoll A, Pintilie G, Hogue CW. A complete small molecule dataset from the protein data bank. FEBS Lett 2006;580(6):1649-53.

16. López G, Valencia A, Tress ML. Firestar-prediction of functionally important residues using structural templates and alignment reliability. Nucleic Acids Res 2007;35:W573-7.

17. Yuan Z, Bailey TL, Teasdale RD. Prediction of protein B-factor profiles. Proteins 2005;58(4):905-12.

18. Parthasarathy S, Murthy MR. Protein thermal stability: Insights from atomic displacement parameters (B values). Protein Eng 2000;13(1):9-13

19. Hinkle A, Tobacman LS. Folding and function of the troponin tail domain. Effects of cardiomyopathic troponin T mutations. J Biol Chem 2003;278(1):506-13

20. Suhre K, Sanejouand YH. EINemo: A normal mode web server for protein movement analysis and the generation of templates for molecular replacement. Nucleic Acids Res 2004;32:W610-4.

21. Langer T, Krovat EM. Chemical feature-based pharmacophores and virtual library screening for discovery of new leads. Curr Opin Drug Discov Devel 2003;6(3):370-6.

22. Wilson RP, Yusuf S. In silico design, docking, synthesis and antimicrobial evaluation of 2,5-disubstituted 1,3,4-oxadiazole derivatives. Int J Pharm Sci Res 2016;7(5):2074-82

23. Oda A, Okayasu M, Kamiyama Y, Yoshida T, Takahashi O, Matsuzaki H. Evaluation of docking accuracy and investigation of roles of parameters and each term in scoring functions for protein-ligand docking using ArgusLab software. Bull Chem Soc Jpn 2007;80(10):1920-25.

24. Kumar A, Ramanathan K. Exploring the structural and functional impact of the ALK F1174L mutation using bioinformatics approach. J Mol Model 2014;20(7):2324.

25. Han LY, Lin HH, Li ZR, Zheng CJ, Cao ZW, Xie B, et al. PEARLS: Program for energetic analysis of receptor-ligand system. J Chem Inf Model 2006;46(1):445-50.

26. Chaitanya M, Babajan B, Anuradha CM, Naveen M, Rajasekhar C, Madhusudana $\mathrm{P}$, et al. Exploring the molecular basis for selective binding of Mycobacterium tuberculosis Asp kinase toward its natural substrates and feedback inhibitors: A docking and molecular dynamics study. J Mol Model 2010;16(8):1357-67.

27. Hess B, Kutzner C, Spoel D, Lindahl E. GROMACS 4: Algorithms for highly efficient, load-balanced, and scalable molecular simulation. J Chem Theory Comput 2008;4(3):435-47.

28. Meagher KL, Carlson HA. Solvation influences flap collapse in HIV-1 protease. Proteins 2005;58(1):119-25.

29. Darden T, Perera L, Li L, Pedersen L. New tricks for modelers from the crystallography toolkit: The particle mesh Ewald algorithm and its use in nucleic acid simulations. Structure 1999;7(3):R55-60.

30. van Gunsteren WF, Berendsen HJ. Algorithms for macromolecular dynamics and constraint dynamics. Mol Phys 1977;34(5):1311-27.

31. Rajasekaran R, George Priya Doss C, Sudandiradoss C, Ramanathan K, Purohit R, Sethumadhavan R. Effect of deleterious nsSNP on the HER2 receptor based on stability and binding affinity with herceptin: A computational approach. C R Biol 2008;331(6):409-17.

32. Chandran D, Pappachen LK, Prathap M, Jinsha MJ, Jilsha G. In silico drug design and molecular docking studies of some novel benzothiazole derivatives as anti-cancer and anti-inflammatory agents. Int J Pharm Pharm Sci 2014;6 Suppl:203-8.

33. Nishtha P, Kant PR, Raj ZN. Comparison of commercially available drugs for type 2 diabetes with natural molecule from tinospora. IJPPS 2014;8(7):173-5.

34. Awad MM, Katayama R, McTigue M, Liu W, Deng YL, Brooun A, et al. Acquired resistance to crizotinib from a mutation in CD74-ROS1. N Engl J Med 2013;368(25):2395-401.

35. Wallace AC, Laskowski RA, Thornton JM. LIGPLOT: A program to generate schematic diagrams of protein-ligand interactions. Protein Eng $1995 ; 8(2): 127-34$ 